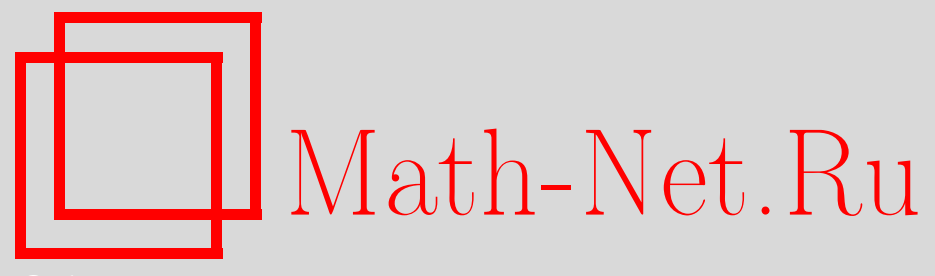

А. К. Уринов, К. Т. Каримов, Задача Дирихле для трехмерного уравнения смешанного типа с тремя сингулярными коэффициентами, Вестн. Сам. гос. техн. ун-та. Сер. Физ.-мат. науки, 2017, номер 4, 665-683

DOI: https://doi.org/10.14498/vsgtu1559

Использование Общероссийского математического портала MathNet.Ru подразумевает, что вы прочитали и согласны с пользовательским соглашением

http: //www . mathnet.ru/rus/agreement

Параметры загрузки:

IP : 54.198 .187 .58

26 апреля 2023 г., 13:39:52

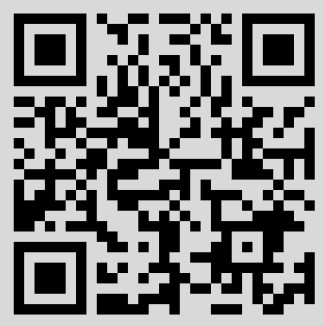


Вестн. Сам. гос. техн. ун-та. Сер. Физ.-мат. науки. 2017. Т. 21, № 4 . С. $665-683$ ISSN: 2310-7081 (online), 1991-8615 (print)

УДК 517.956.6

\title{
Задача Дирихле для трехмерного уравнения смешанного типа с тремя сингулярными коэффициентами
}

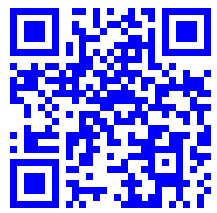

\section{А. К. Уринов, К. Т. Каримов}

Ферганский государственный университет, Узбекистан, 712000, Фергана, ул. Мураббийлар, 19.

\begin{abstract}
Аннотация
Рассматривается трехмерное уравнения смешанного типа с тремя сингулярными коэффициентами, для которого в параллелепипеде исследуется задача Дирихле. Исследование поставленной задачи проводится с помощью метода разделения переменных Фурье и спектрального анализа. Для поставленной задачи с помощью метода Фурье получены две одномерные спектральные задачи. На основании свойства полноты систем собственных функций этих задач доказана теорема единственности. Решение исследуемой задачи построено в виде суммы двойного ряда Фурье-Бесселя. В обосновании равномерной сходимости построенного ряда использовались асимптотические оценки функций Бесселя действительного и мнимого аргумента. На их основе получены оценки для каждого члена ряда, которые позволили доказать сходимость полученного ряда и его производных до второго порядка включительно, а также теорему существования в классе регулярных решений.
\end{abstract}

Ключевые слова: задача Дирихле, уравнения смешанного типа, спектральный метод, единственность решения, существование решения.

Получение: 19 июля 2017 г. / Исправление: 30 ноября 2017 г. Принятие: 18 декабря 2017 г. / Публикация онлайн: 22 декабря 2017 г.

\section{Научная статья}

(2)(7) Контент публикуется на условиях лицензии Creative Commons Attribution 4.0 International (https://creativecommons.org/licenses/by/4.0/deed.ru)

\section{Образец для цитирования}

У р и н о А. К., К ар и м о в К. Т. Задача Дирихле для трехмерного уравнения смешанного типа с тремя сингулярными коэффициентами // Вестн. Сам. гос. техн. ун-та. Сер. Физ.-мат. науки, 2017. Т. 21, № 4. С. 665-683. doi:10.14498/vsgtu1559.

\section{Сведения об авторах}

Ахмаджон Кушакович Уринов (10) http://orcid.org/0000-0002-9586-1799

доктор физико-математических наук, профессор; профессор; каф. математического анализа и дифференциальных уравнений; e-mail: urinovak@mail.ru

Камолиддин Туйчибаевич Каримов (1) http://orcid.org/0000-0002-9098-4116

кандидат физико-математических наук; докторант; каф. математического анализа и дифференциальных уравнений; e-mail: karimovk80@mail.ru 
Постановка задачи. Задача Дирихле для уравнений смешанного типа $[1,2]$ является не всегда корректно поставленной в смысле Адамара. ${ }^{1}$ Поэтому поиск смешанных областей, в которых задача Дирихле была бы корректно поставленной, представляет интерес для многих исследователей. Многочисленные работы (см., например, библиографию к [3], а также работы [4-10], опубликованные в последнее время), посвященные данной проблеме, преимущественно затрагивают двумерные задачи. Трехмерные задачи рассматриваются в работах [11-14], при этом задача Дирихле для трехмерных уравнений смешанного типа с сингулярными коэффициентами до сих пор остается малоизученной.

В настоящей работе рассматривается трехмерное уравнения смешанного типа с тремя сингулярными коэффициентами:

$$
u_{x x}+(\operatorname{sgn} y) u_{y y}+u_{z z}+\frac{2 \alpha}{x} u_{x}+\frac{2 \beta}{|y|} u_{y}+\frac{2 \gamma}{z} u_{z}=0
$$

для которого задача Дирихле исследуется в параллелепипеде

$$
\Omega=\{(x, y, z): 0<x<1,-a<y<b, 0<z<1\}, \quad a>0, \quad b>0 .
$$

Здесь $u=u(x, y, z)$ - неизвестная функция; параметры $\alpha, \beta, \gamma \in \mathbb{R} \backslash\{0\}$, причем $-\infty<\alpha<1 / 2,-1 / 2<\beta<1 / 2,-\infty<\gamma<1 / 2$.

Уравнение (1) в области $\Omega$ принадлежит смешанному типу: уравнение в области $\Omega^{+}=\Omega \cap\{y>0\}$ принадлежит эллиптическому типу, а в области $\Omega^{-}=\Omega \cap\{y<0\}-$ гиперболическому типу. Плоскости $x=0, y=0$ и $z=0$ являются плоскостями сингулярности коэффициентов уравнения. Среди них $y=0-$ плоскость изменения типа уравнения.

Рассмотрим следующую задачу и исследуем ее однозначную разрешимость.

ЗАДАчА D. Найти функиию $u(x, y, z) \in C(\bar{\Omega}) \cap C^{2}\left(\Omega^{+} \cup \Omega^{-}\right)$, удовлетворяюшую в области $\Omega^{+} \cup \Omega^{-}$уравнению $(1)$, краевым условиям

$$
\begin{gathered}
u(0, y, z)=u(1, y, z)=0, \quad-a \leqslant y \leqslant b, \quad 0 \leqslant z \leqslant 1 ; \\
u(x, y, 0)=u(x, y, 1)=0, \quad-a \leqslant y \leqslant b, \quad 0 \leqslant x \leqslant 1 ; \\
u(x, b, z)=f(x, z), \quad 0 \leqslant x \leqslant 1, \quad 0 \leqslant z \leqslant 1 ; \\
u(x,-a, z)=g(x, z), \quad 0 \leqslant x \leqslant 1, \quad 0 \leqslant z \leqslant 1,
\end{gathered}
$$

и условию склеивания

$$
\lim _{y \rightarrow-0}(-y)^{2 \beta} u_{y}(x, y, z)=\lim _{y \rightarrow+0} y^{2 \beta} u_{y}(x, y, z), \quad 0<x<1, \quad 0<z<1,
$$

где $f(x, z), g(x, z)$ - заданнье непрерьвные фбункиии.

\footnotetext{
${ }^{1}$ Во многих работах отмечается, что интерес к задаче Дирихле для уравнений смешанного типа возник после работы Ф. И. Франкля [1], где впервые обращено внимание на то, что задачи трансзвуковой газовой динамики сводятся к уравнениям этого типа, а А. В. Бицадзе в работе [2] показал, что задача Дирихле для уравнения $u_{x x}+(\operatorname{sgn} y) u_{y y}=0$ поставлена некорректно.
} 
1. Построение собственных функций. Сначала найдем нетривиальные решения задачи (1), (2), (3), (6). С этой целью, разделив переменные по формуле $u(x, y, z)=W(x, z) Q(y)$, из уравнения (1) получим

$$
\begin{gathered}
(\operatorname{sgn} y) Q^{\prime \prime}(y)+\frac{2 \beta}{|y|} Q^{\prime}(y)-\lambda Q(y)=0, \quad-a<y<b \\
W_{x x}+W_{z z}+\frac{2 \alpha}{x} W_{x}+\frac{2 \gamma}{z} W_{z}+\lambda W=0, \quad 0<x<1, \quad 0<z<1,
\end{gathered}
$$

где $\lambda$ - константа разделения.

Учитывая однородные краевые условия (2) и (3), для уравнения (8) получим задачу на собственные значения в квадрате $\Pi=\{(x, z): 0 \leqslant x \leqslant 1$, $0 \leqslant z \leqslant 1\}$ со следующими краевыми условиями:

$$
\begin{array}{ll}
W(0, z)=W(1, z)=0, & 0 \leqslant z \leqslant 1 \\
W(x, 0)=W(x, 1)=0, & 0 \leqslant x \leqslant 1 .
\end{array}
$$

Путем разделения переменных $W(x, z)=X(x) Z(z)$ эта задача сводится к двум задачам на собственные значения:

$$
\begin{gathered}
X^{\prime \prime}(x)+\frac{2 \alpha}{x} X^{\prime}(x)+\mu X(x)=0, \\
X(0)=0, \quad X(1)=0 ; \\
Z^{\prime \prime}(z)+\frac{2 \gamma}{z} Z^{\prime}(z)+(\lambda-\mu) Z(z)=0, \\
Z(0)=0, \quad Z(1)=0,
\end{gathered}
$$

где $\mu$ - константа разделения.

Из уравнения (9), произведя замену

$$
X(x)=(t / \sqrt{\mu})^{1 / 2-\alpha} p(t),
$$

где $t=\sqrt{\mu} x$, получим уравнение Бесселя $[15, \S 3.1]$ :

$$
t^{2} p^{\prime \prime}(t)+t p^{\prime}(t)+\left(t^{2}-(1 / 2-\alpha)^{2}\right) p(t)=0 .
$$

Принимая во внимание вид общего решения [15, § 3.1] уравнения (13) и введенные обозначения, получим общее решение уравнения (9) в виде

$$
X(x)=d_{1} x^{1 / 2-\alpha} J_{1 / 2-\alpha}(\sqrt{\mu} x)+d_{2} x^{1 / 2-\alpha} Y_{1 / 2-\alpha}(\sqrt{\mu} x) .
$$

Здесь $d_{1}$ и $d_{2}$ - произвольные постоянные, $J_{l}(x)$ и $Y_{l}(x)$ - функция Бесселя порядка $l$ первого и второго рода $[15, \S \S 3.1,3.5]$ соответственно. Из (14) следует, что решение уравнения (9), удовлетворяющее первому из условий (10), существует при $\alpha<1 / 2$ и оно определяется равенством

$$
X(x)=d_{1} x^{1 / 2-\alpha} J_{1 / 2-\alpha}(\sqrt{\mu} x) .
$$

Подставляя (15) во второе из условий (10), получим условия существования нетривиального решения задачи (9), (10):

$$
J_{1 / 2-\alpha}(\sqrt{\mu})=0 .
$$


Известно, что при $l>-1$ функция Бесселя $J_{l}(z)$ имеет счетное число нулей, причем все они вещественны и с попарно противоположными знаками [15, § 15.23]. Так как $1 / 2-\alpha>0$, уравнение (16) имеет счетное число вещественных корней. Обозначая через $\sigma_{n}-n$-ный положительный корень уравнения (16), получим значения параметра $\mu$, при которых существуют нетривиальные решения задачи (9),(10), т. е. ее собственные значения:

$$
\mu_{n}=\sigma_{n}^{2}, \quad n \in \mathbb{N}
$$

Полагая в $(15) \mu=\mu_{n}, n \in \mathbb{N}$ и $d_{1}=1$, получим нетривиальные решения (собственные функции) задачи (9), (10):

$$
X_{n}(x)=x^{1 / 2-\alpha} J_{1 / 2-\alpha}\left(\sigma_{n} x\right), \quad n \in \mathbb{N}
$$

Теперь перейдем к исследованию задачи (11), (12). Методом, примененным при решении задачи (9), (10), найдем общее решение уравнения (11) в виде

$$
Z(z)=d_{3} z^{1 / 2-\gamma} J_{1 / 2-\gamma}\left(\sqrt{\lambda-\mu_{n}} z\right)+d_{4} z^{1 / 2-\gamma} Y_{1 / 2-\gamma}\left(\sqrt{\lambda-\mu_{n}} z\right)
$$

где $d_{3}$ и $d_{4}$ - произвольные постоянные. Подставляя (18) в краевые условия (12), имеем $d_{4}=0$ и

$$
J_{1 / 2-\gamma}\left(\sqrt{\lambda-\mu_{n}}\right)=0
$$

Учитывая, что $\gamma<1 / 2$ и обозначая через $\delta_{m}-m$-ный положительный корень уравнения (19), получим значения параметра $\lambda$, при которых существуют нетривиальные решения задачи $(11),(12)$ :

$$
\lambda_{n m}=\sigma_{n}^{2}+\delta_{m}^{2}, \quad n, m \in \mathbb{N}
$$

Полагая в (18) $\lambda=\lambda_{n m}, n, m \in \mathbb{N}, d_{3}=1$ и $d_{4}=0$, получим нетривиальные решения (собственные функции) задачи (11), (12) в виде

$$
Z_{m}(z)=z^{1 / 2-\gamma} J_{1 / 2-\gamma}\left(\delta_{m} z\right), \quad m \in \mathbb{N}
$$

Полагая в уравнении (7) $\lambda=\lambda_{n m}$, найдем общее решения этого уравнения при $y>0$ и $y<0$ :

$$
Q_{n m}(y)=\left\{\begin{array}{c}
a_{n m} y^{1 / 2-\beta} I_{1 / 2-\beta}\left(\sqrt{\lambda_{n m}} y\right)+ \\
\quad+b_{n m} y^{1 / 2-\beta} K_{1 / 2-\beta}\left(\sqrt{\lambda_{n m}} y\right), \quad y>0 ; \\
c_{n m}(-y)^{1 / 2-\beta} J_{1 / 2-\beta}\left[\sqrt{\lambda_{n m}}(-y)\right]+ \\
+d_{n m}(-y)^{1 / 2-\beta} Y_{1 / 2-\beta}\left[\sqrt{\lambda_{n m}}(-y)\right], \quad y<0 .
\end{array}\right.
$$

Здесь $n, m \in \mathbb{N} ; a_{n m}, b_{n m}, c_{n m}$ и $d_{n m}$ - произвольные постоянные, $I_{\omega}(z)$ и $K_{\omega}(z)$ - модифицированные функции Бесселя порядка $\omega$ первого и третьего рода $[15, \S 3.7]$ соответственно. 
Подставляя $(17),(20),(21)$ в равенство $u(x, y, z)=X(x) Q(y) Z(z)$, получим нетривиальные в области $\Omega$ решения уравнения $(1)$, удовлетворяющие условиям (2) и (3):

$$
u_{n m}=\left\{\begin{aligned}
& x^{1 / 2-\alpha} y^{1 / 2-\beta} z^{1 / 2-\gamma} J_{1 / 2-\alpha}\left(\sigma_{n} x\right) J_{1 / 2-\gamma}\left(\delta_{m} z\right) \times \\
& \times\left(a_{n m} I_{1 / 2-\beta}\left(\sqrt{\lambda_{n m}} y\right)+b_{n m} K_{1 / 2-\beta}\left(\sqrt{\lambda_{n m}} y\right)\right), \quad y>0 ; \\
& x^{1 / 2-}-\alpha(-y)^{1 / 2-\beta} z^{1 / 2-\gamma} J_{1 / 2-\alpha}\left(\sigma_{n} x\right) J_{1 / 2-\gamma}\left(\delta_{m} z\right) \times \\
& \times\left(c_{n m} J_{1 / 2-\beta}\left[\sqrt{\lambda_{n m}}(-y)\right]+d_{n m} Y_{1 / 2-\beta}\left[\sqrt{\lambda_{n m}}(-y)\right]\right), \quad y<0 .
\end{aligned}\right.
$$

Теперь подберем постоянные $a_{n m}, b_{n m}, c_{n m}$ и $d_{n m}$ так, чтобы для функций $u_{n m}(x, y, z)$ выполнялись условия склеивания (6) и условие

$$
u(x,-0, z)=u(x,+0, z) .
$$

Непосредственной проверкой нетрудно убедиться, что равенство (22) выполняется, если $d_{n m}=-\left(\pi b_{n m}\right) / 2$ при любых $a_{n m}$ и $c_{n m}$, а условия склеивания (6) выполняются, если

$$
c_{n m}=\pi b_{n m} \operatorname{tg}(\pi(1+2 \beta) / 4) / 2-a_{n m}, \quad d_{n m}=-\left(\pi b_{n m}\right) / 2 .
$$

Тогда на основании этих равенств система функций $u_{n m}(x, y, z)$ запишется в следующем виде:

$$
\begin{aligned}
& u_{n m}(x, y, z)= \\
& =\left\{\begin{array}{r}
x^{1 / 2-}-\alpha y^{1 / 2-\beta} z^{1 / 2-\gamma} J_{1 / 2-\alpha}\left(\sigma_{n} x\right) J_{1 / 2-\gamma}\left(\delta_{m} z\right) \times \\
\times\left(a_{n m} I_{1 / 2-\beta}\left(\sqrt{\lambda_{n m}} y\right)+b_{n m} K_{1 / 2-\beta}\left(\sqrt{\lambda_{n m}} y\right)\right), y>0 \\
x^{1 / 2-}-\alpha(-y)^{1 / 2-\beta} z^{1 / 2-\gamma} J_{1 / 2-\alpha}\left(\sigma_{n} x\right) J_{1 / 2-\gamma}\left(\delta_{m} z\right) \times \\
\times\left(-a_{n m} J_{1 / 2-\beta}\left[\sqrt{\lambda_{n m}}(-y)\right]+b_{n m} \bar{Y}_{1 / 2-\beta}\left[\sqrt{\lambda_{n m}}(-y)\right]\right), y<0
\end{array}\right.
\end{aligned}
$$

где

$$
\bar{Y}_{1 / 2-\beta}\left[\sqrt{\lambda_{n m}}(-y)\right]=\frac{\pi}{2 \cos (\beta \pi)}\left(J_{1 / 2-\beta}\left[\sqrt{\lambda_{n m}}(-y)\right]+J_{\beta-1 / 2}\left[\sqrt{\lambda_{n m}}(-y)\right]\right) .
$$

\section{2. Единственность решения задачи D.}

Теорема 1. Если существует решение и $(x, y, z)$ задачи $D$, то оно единственно тогда и только тогда, когда

$$
\begin{aligned}
\Delta_{n m}(a, b)=I_{1 / 2-\beta}\left(\sqrt{\lambda_{n m}} b\right) \bar{Y}_{1 / 2-\beta}\left(\sqrt{\lambda_{n m}} a\right)+ & \\
& +K_{1 / 2-\beta}\left(\sqrt{\lambda_{n m}} b\right) J_{1 / 2-\beta}\left(\sqrt{\lambda_{n m}} a\right) \neq 0
\end{aligned}
$$

при всех $n, m \in \mathbb{N}$.

Доказательств о. Пусть $u(x, y, z)$ - решение задачи D. Рассмотрим следующую функцию:

$$
u_{n m}(y)=\int_{0}^{1} \int_{0}^{1} u(x, y, z) x^{2 \alpha} X_{n}(x) z^{2 \gamma} Z_{m}(z) d x d z=
$$




$$
=\int_{0}^{1}\left(\int_{0}^{1} u(x, y, z) x^{1 / 2+\alpha} J_{1 / 2-\alpha}\left(\sigma_{n} x\right) d x\right) z^{1 / 2+\gamma} J_{1 / 2-\gamma}\left(\delta_{m} z\right) d z,
$$

где $m, n \in \mathbb{N}$.

Согласно [15, §15.25], система функций $\left\{J_{1 / 2-\alpha}\left(\sigma_{n} x\right)\right\}_{n=1}^{\infty}$ ортогональна с весом $x$ на отрезке $[0,1]$. Поэтому в силу равенства

$$
\begin{aligned}
\int_{0}^{1} x^{1 / 2-\alpha} J_{1 / 2-\alpha}\left(\sigma_{m} x\right) x^{1 / 2-\alpha} J_{1 / 2-\alpha} & \left(\sigma_{n} x\right) x^{2 \alpha} d x= \\
& =\int_{0}^{1} x J_{1 / 2-\alpha}\left(\sigma_{m} x\right) J_{1 / 2-\alpha}\left(\sigma_{n} x\right) d x=0
\end{aligned}
$$

система собственных функций (17) ортогональна с весом $x^{2 \alpha}$ на $[0,1]$.

Согласно $[15, \S 18.1]$, система функций $\left\{J_{1 / 2-\alpha}\left(\sigma_{n} x\right)\right\}_{n=1}^{\infty}$ полна с весом $x$ в пространстве $L_{2}[0,1]$ и имеет место соотношение

$$
\int_{0}^{1} x J_{1 / 2-\alpha}^{2}\left(\sigma_{n} x\right) d x=\frac{1}{2} J_{3 / 2-\alpha}^{2}\left(\sigma_{n}\right) .
$$

Отсюда следует, что система собственных функций (17) полна в пространстве $L_{2}[0,1]$ с весом $x^{2 \alpha}$ и для функций этой системы имеет место соотношение

$$
\int_{0}^{1} x^{1-2 \alpha} J_{1 / 2-\alpha}^{2}\left(\sigma_{n} x\right) x^{2 \alpha} d x=\int_{0}^{1} x J_{1 / 2-\alpha}^{2}\left(\sigma_{n} x\right) d x=\frac{1}{2} J_{3 / 2-\alpha}^{2}\left(\sigma_{n}\right) .
$$

Проведя те же рассуждения, можно убедиться в том, что система собственных функций (20) полна в $L_{2}[0,1]$ с весом $z^{2 \gamma}$.

На основании (24) введем функции

$$
u_{n m}^{\varepsilon_{1} \varepsilon_{2}}(y)=\int_{\varepsilon_{1}}^{1-\varepsilon_{1}} \int_{\varepsilon_{2}}^{1-\varepsilon_{2}} u(x, y, z) x^{2 \alpha} X_{n}(x) z^{2 \gamma} Z_{m}(z) d x d z \quad n, m \in \mathbb{N},
$$

где $\varepsilon_{1}$ и $\varepsilon_{2}$ - достаточно малые положительные числа. Очевидно,

$$
\lim _{\substack{\varepsilon_{1} \rightarrow 0 \\ \varepsilon_{2} \rightarrow 0}} u_{n m}^{\varepsilon_{1} \varepsilon_{2}}(y)=u_{n m}(y) .
$$

Продифференцируем равенство (25) по $y$ при $y \in(-a, 0) \cup(0, b)$ :

$$
\begin{aligned}
& {\left[u_{n m}^{\varepsilon_{1} \varepsilon_{2}}(y)\right]^{\prime}=\int_{\varepsilon_{1}}^{1-\varepsilon_{1}} \int_{\varepsilon_{2}}^{1-\varepsilon_{2}} u_{y}(x, y, z) x^{2 \alpha} X_{n}(x) z^{2 \gamma} Z_{m}(z) d x d z, \quad n, m \in \mathbb{N} ;} \\
& {\left[u_{n m}^{\varepsilon_{1} \varepsilon_{2}}(y)\right]^{\prime \prime}=\int_{\varepsilon_{1}}^{1-\varepsilon_{1}} \int_{\varepsilon_{2}}^{1-\varepsilon_{2}} u_{y y}(x, y, z) x^{2 \alpha} X_{n}(x) z^{2 \gamma} Z_{m}(z) d x d z, \quad n, m \in \mathbb{N} .}
\end{aligned}
$$

Учитывая уравнение (1), из последних равенств имеем

$$
\begin{array}{r}
{\left[u_{n m}^{\varepsilon_{1} \varepsilon_{2}}(y)\right]^{\prime \prime}=-(\operatorname{sgn} y) \int_{\varepsilon_{1}}^{1-\varepsilon_{1}} \int_{\varepsilon_{2}}^{1-\varepsilon_{2}}\left(u_{x x}+\frac{2 \alpha}{x} u_{x}+u_{z z}+\frac{2 \gamma}{z} u_{z}+\frac{2 \beta}{|y|} u_{y}\right) \times} \\
\times x^{2 \alpha} X_{n}(x) z^{2 \gamma} Z_{m}(z) d x d z=
\end{array}
$$


$=-(\operatorname{sgn} y)\left[\int_{\varepsilon_{2}}^{1-\varepsilon_{2}}\left(\int_{\varepsilon_{1}}^{1-\varepsilon_{1}} u_{x x} x^{2 \alpha} X_{n}(x) d x+\right.\right.$

$$
\left.+\int_{\varepsilon_{1}}^{1-\varepsilon_{1}} \frac{2 \alpha}{x} u_{x} x^{2 \alpha} X_{n}(x) d x\right) z^{2 \gamma} Z_{m}(z) d z+
$$

$$
\begin{aligned}
& +\int_{\varepsilon_{1}}^{1-\varepsilon_{1}}\left(\int_{\varepsilon_{2}}^{1-\varepsilon_{2}} u_{z z} z^{2 \gamma} Z_{m}(z) d z+\right. \\
& \left.\left.\quad+\int_{\varepsilon_{2}}^{1-\varepsilon_{2}} \frac{2 \gamma}{z} u_{z} z^{2 \gamma} Z_{m}(z) d z\right) x^{2 \alpha} X_{n}(x) d z+\frac{2 \beta}{|y|}\left[u_{n m}^{\varepsilon_{1} \varepsilon_{2}}(y)\right]^{\prime}\right] .
\end{aligned}
$$

Рассмотрим следующие интегралы:

$$
\begin{array}{r}
\int_{\varepsilon_{1}}^{1-\varepsilon_{1}} u_{x x} x^{2 \alpha} X_{n}(x) d x=\int_{\varepsilon_{1}}^{1-\varepsilon_{1}} x^{2 \alpha} X_{n}(x) d u_{x}= \\
=\left.u_{x} x^{2 \alpha} X_{n}(x)\right|_{x=\varepsilon_{1}} ^{x=1-\varepsilon_{1}}-\int_{\varepsilon_{1}}^{1-\varepsilon_{1}} u_{x}\left[x^{2 \alpha} X_{n}(x)\right]^{\prime} d x= \\
=\left.u_{x} x^{2 \alpha} X_{n}(x)\right|_{x=\varepsilon_{1}} ^{x=1-\varepsilon_{1}}-\left.u\left[x^{2 \alpha} X_{n}(x)\right]^{\prime}\right|_{x=\varepsilon_{1}} ^{x=1-\varepsilon_{1}}+\int_{\varepsilon_{1}}^{1-\varepsilon_{1}} u\left[x^{2 \alpha} X_{n}(x)\right]^{\prime \prime} d x= \\
=\left.u_{x} x^{2 \alpha} X_{n}(x)\right|_{x=\varepsilon_{1}} ^{x=1-\varepsilon_{1}}-\left.u\left[x^{2 \alpha} X_{n}(x)\right]^{\prime}\right|_{x=1-\varepsilon_{1}} ^{x=\varepsilon_{1}}+ \\
+\int_{\varepsilon_{1}}^{1-\varepsilon_{1}} u x^{2 \alpha}\left[X_{n}^{\prime \prime}(x)+2 \frac{2 \alpha}{x} X_{n}^{\prime}(x)+\left(4 \alpha^{2}-2 \alpha\right) x^{-2} X_{n}(x)\right] d x ;
\end{array}
$$

$$
\begin{aligned}
& \int_{\varepsilon_{1}}^{1-\varepsilon_{1}} \frac{2 \alpha}{x} u_{x} x^{2 \alpha} X_{n}(x) d x=\int_{\varepsilon_{1}}^{1-\varepsilon_{1}} 2 \alpha x^{2 \alpha-1} X_{n}(x) d u= \\
& =\left.2 \alpha x^{2 \alpha-1} X_{n}(x) u\right|_{x=\varepsilon_{1}} ^{x=1-\varepsilon_{1}}-2 \alpha \int_{\varepsilon_{1}}^{1-\varepsilon_{1}} u\left[x^{2 \alpha-1} X_{n}(x)\right]^{\prime} d x=
\end{aligned}
$$

$=\left.2 \alpha x^{2 \alpha-1} X_{n}(x) u\right|_{x=\varepsilon_{1}} ^{x=1-\varepsilon_{1}}-\int_{\varepsilon_{1}}^{1-\varepsilon_{1}} u x^{2 \alpha}\left[\frac{2 \alpha}{x} X_{n}^{\prime}(x)+\left(4 \alpha^{2}-2 \alpha\right) x^{-2} X_{n}(x)\right] d x$.

На основании полученных выше равенств имеем

$$
\begin{aligned}
& \int_{\varepsilon_{1}}^{1-\varepsilon_{1}} u_{x x} x^{2 \alpha} X_{n}(x) d x+\int_{\varepsilon_{1}}^{1-\varepsilon_{1}} \frac{2 \alpha}{x} u_{x} x^{2 \alpha} X_{n}(x) d x= \\
& =\left.\left[u_{x} X_{n}(x)-u X_{n}^{\prime}(x)\right] x^{2 \alpha}\right|_{x=\varepsilon_{1}} ^{x=1-\varepsilon_{1}}+\int_{\varepsilon_{1}}^{1-\varepsilon_{1}} u x^{2 \alpha}\left[X_{n}^{\prime \prime}(x)+\frac{2 \alpha}{x} X_{n}^{\prime}(x)\right] d x= \\
& =\left.\left[u_{x} X_{n}(x)-u X_{n}^{\prime}(x)\right] x^{2 \alpha}\right|_{x=\varepsilon_{1}} ^{x=1-\varepsilon_{1}}-\sigma_{n}^{2} \int_{\varepsilon_{1}}^{1-\varepsilon_{1}} u x^{2 \alpha} X_{n}(x) d x .
\end{aligned}
$$




\section{Аналогично находим}

$$
\begin{gathered}
\int_{\varepsilon_{2}}^{1-\varepsilon_{2}} u_{z z} z^{2 \gamma} Z_{m}(z) d z+\int_{\varepsilon_{2}}^{1-\varepsilon_{2}} \frac{2 \gamma}{z} u_{z} z^{2 \gamma} Z_{m}(z) d z= \\
=\left.\left[u_{z} Z_{m}(z)-u Z_{m}^{\prime}(z)\right] z^{2 \gamma}\right|_{z=\varepsilon_{2}} ^{z=1-\varepsilon_{2}}-\delta_{m}^{2} \int_{\varepsilon_{2}}^{1-\varepsilon_{2}} u z^{2 \gamma} Z_{m}(z) d z .
\end{gathered}
$$

Подставляя (28) и (29) в равенство (27), получим

$$
\begin{gathered}
{\left[u_{n m}^{\varepsilon_{1} \varepsilon_{2}}(y)\right]^{\prime \prime}=-(\operatorname{sgn} y)\left[\int _ { \varepsilon _ { 2 } } ^ { 1 - \varepsilon _ { 2 } } \left(\left.\left[u_{x} X_{n}(x)-u X_{n}^{\prime}(x)\right] x^{2 \alpha}\right|_{x=\varepsilon_{1}} ^{x=1-\varepsilon_{1}}-\right.\right.} \\
\left.-\sigma_{n}^{2} \int_{\varepsilon_{1}}^{1-\varepsilon_{1}} u x^{2 \alpha} X_{n}(x) d x\right) z^{2 \gamma} Z_{m}(z) d z+ \\
+\int_{\varepsilon_{1}}^{1-\varepsilon_{1}}\left(\left.\left[u_{z} Z_{m}(z)-u Z_{m}^{\prime}(z)\right] z^{2 \gamma}\right|_{z=\varepsilon_{2}} ^{z=1-\varepsilon_{2}}-\right. \\
\left.\left.-\delta_{m}^{2} \int_{\varepsilon_{2}}^{1-\varepsilon_{2}} u z^{2 \gamma} Z_{m}(z) d z\right) x^{2 \alpha} X_{n}(x) d x+\frac{2 \beta}{|y|}\left[u_{n m}^{\varepsilon_{1} \varepsilon_{2}}(y)\right]^{\prime}\right] .
\end{gathered}
$$

Предварительно заметим, что нижеследующие пределы конечны:

$$
\lim _{x \rightarrow+0} x^{2 \alpha} X_{n}^{\prime}(x)=\frac{2^{1 / 2+\alpha} \sigma_{n}^{1 / 2-\alpha}}{\Gamma[1 / 2-\alpha]}, \quad \lim _{z \rightarrow+0} z^{2 \gamma} Z_{m}^{\prime}(z)=\frac{2^{1 / 2+\gamma} \delta_{m}^{1 / 2-\gamma}}{\Gamma[1 / 2-\gamma]} .
$$

Отсюда, требуя

$$
\lim _{x \rightarrow 1-0} X_{n}(x) u_{x}=0, \quad \lim _{z \rightarrow 1-0} Z_{m}(z) u_{z}=0,
$$

а затем переходя к пределу при $\varepsilon_{1} \rightarrow 0, \varepsilon_{2} \rightarrow 0$ и учитывая граничные условия $(2),(3),(10),(12)$ и равенство $(26)$, получим, что $u_{n m}(y)$ удовлетворяет дифференциальному уравнению

$$
(\operatorname{sgn} y) u_{n m}^{\prime \prime}(y)+\frac{2 \beta}{|y|} u_{n m}^{\prime}(y)-\lambda_{n m} u_{n m}(y)=0, \quad-a<y<b,
$$

т. е. уравнению (7). Следовательно, $u_{n m}(y)=Q_{n m}(y)$.

Теперь из $(24)$ находим $u_{n m}(b)$ и $u_{n m}(-a)$ :

$$
\begin{gathered}
u_{n m}(b)=\int_{0}^{1} \int_{0}^{1} u(x, b, z) x^{2 \alpha} X_{n}(x) z^{2 \gamma} Z_{m}(z) d x d z= \\
=\int_{0}^{1} \int_{0}^{1} f(x, y) x^{2 \alpha} X_{n}(x) z^{2 \gamma} Z_{m}(z) d x d z=F_{n m}, \\
u_{n m}(-a)=\int_{0}^{1} \int_{0}^{1} u(x,-a, z) x^{2 \alpha} X_{n}(x) z^{2 \gamma} Z_{m}(z) d x d z= \\
=\int_{0}^{1} \int_{0}^{1} g(x, y) x^{2 \alpha} X_{n}(x) z^{2 \gamma} Z_{m}(z) d x d z=G_{n m} .
\end{gathered}
$$


Принимая во внимание равенства $(30),(31)$ и равенства $d_{n m}=-\left(\pi b_{n m}\right) / 2$, $c_{n m}=b_{n m} \pi \operatorname{tg}[\pi(1+2 \beta) / 4] / 2-a_{n m}$, из $(21)$ находим

$$
\left\{\begin{aligned}
a_{n m} I_{1 / 2-\beta}\left(\sqrt{\lambda_{n m}} b\right)+b_{n m} K_{1 / 2-\beta}\left(\sqrt{\lambda_{n m}} b\right) & =F_{n m} b^{\beta-1 / 2} \\
-a_{n m} J_{1 / 2-\beta}\left(\sqrt{\lambda_{n m}} a\right)+b_{n m} \bar{Y}_{1 / 2-\beta}\left(\sqrt{\lambda_{n m}} a\right) & =G_{n m} a^{\beta-1 / 2}
\end{aligned}\right.
$$

Определитель системы (32) $\Delta_{n m}(a, b)$ имеет вид $(23)$. Так как $\Delta_{n m}(a, b) \neq 0$, система (32) имеет единственное решение:

$$
\begin{aligned}
a_{n m} & =\frac{F_{n m} b^{\beta-1 / 2} \bar{Y}_{1 / 2-\beta}\left(\sqrt{\lambda_{n m}} a\right)-G_{n m} a^{\beta-1 / 2} K_{1 / 2-\beta}\left(\sqrt{\lambda_{n m}} b\right)}{\Delta_{n m}(a, b)}, \\
b_{n m} & =\frac{F_{n m} b^{\beta-1 / 2} J_{1 / 2-\beta}\left(\sqrt{\lambda_{n m}} a\right)+G_{n m} a^{\beta-1 / 2} I_{1 / 2-\beta}\left(\sqrt{\lambda_{n m}} b\right)}{\Delta_{n m}(a, b)},
\end{aligned}
$$

а функции $u_{n m}(y)$ примут следующий вид:

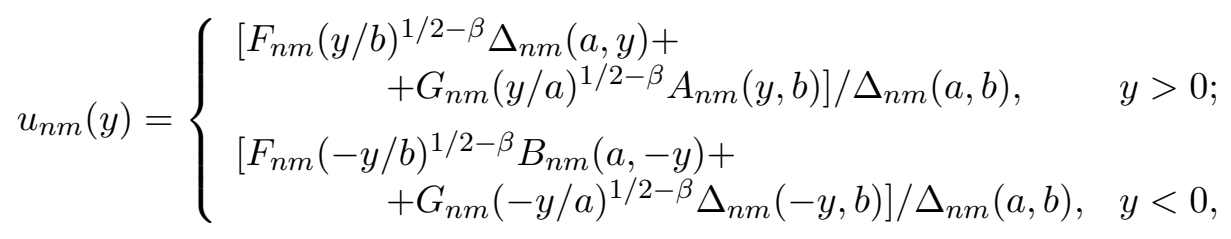

где

$$
\begin{aligned}
& A_{n m}(y, b)=I_{1 / 2-\beta}\left(\sqrt{\lambda_{n m}} b\right) K_{1 / 2-\beta}\left(\sqrt{\lambda_{n m}} y\right)- \\
& -K_{1 / 2-\beta}\left(\sqrt{\lambda_{n m}} b\right) I_{1 / 2-\beta}\left(\sqrt{\lambda_{n m}} y\right), \\
& B_{n m}(a,-y)=J_{1 / 2-\beta}\left(\sqrt{\lambda_{n m}} a\right) \bar{Y}_{1 / 2-\beta}\left(\sqrt{\lambda_{n m}}(-y)\right)- \\
& -\bar{Y}_{1 / 2-\beta}\left(\sqrt{\lambda_{n m}} a\right) J_{1 / 2-\beta}\left(\sqrt{\lambda_{n m}}(-y)\right) .
\end{aligned}
$$

Пусть $f(x, z) \equiv 0$ и $g(x, z) \equiv 0$. Тогда из равенств $(30),(31)$ и (33) следует, что $u_{n m}(y) \equiv 0$, и на основании равенства $(24)$ имеем

$$
\int_{0}^{1}\left(\int_{0}^{1} x^{2 \alpha} z^{2 \gamma} u(x, y, z) x^{1 / 2-\alpha} J_{1 / 2-\alpha}\left(\sigma_{n} x\right) d x\right) z^{1 / 2-\gamma} J_{1 / 2-\gamma}\left(\delta_{m} z\right) d z=0 .
$$

Отсюда в силу полноты системы функций $\left\{z^{1 / 2-\gamma} J_{1 / 2-\gamma}\left(\delta_{m} z\right)\right\}_{m=1}^{\infty}$ с весом $z^{2 \gamma}$ в пространстве $L_{2}[0,1]$ следует, что

$$
\int_{0}^{1} x^{2 \alpha} z^{2 \gamma} u(x, y, z) x^{1 / 2-\alpha} J_{1 / 2-\alpha}\left(\sigma_{n} x\right) d x=0
$$

В силу полноты системы функций $\left\{x^{1 / 2-\alpha} J_{1 / 2-\alpha}\left(\sigma_{n} x\right)\right\}_{n=1}^{\infty}$ с весом $x^{2 \alpha}$ в пространстве $L_{2}[0,1]$ из последнего равенства имеем, что $u(x, y, z) \equiv 0$ для 
всех $x \in[0,1]$ и при любом $y \in[-a, b], z \in[0,1]$. Отсюда следует утверждение теоремы.

3. Существование решения задачи D. Решение задачи Дирихле в области $\Omega$ будем искать в виде

$$
u(x, y, z)=\sum_{n=1}^{\infty} \sum_{m=1}^{\infty} u_{n m}(x, y, z)= \begin{cases}\sum_{n=1}^{\infty} \sum_{m=1}^{\infty} u_{n m}^{+}(x, y, z), & y>0 \\ \sum_{n=1}^{\infty} \sum_{m=1}^{\infty} u_{n m}^{-}(x, y, z), & y<0\end{cases}
$$

где

$$
\begin{gathered}
u_{n m}^{+}(x, y, z)=x^{1 / 2-\alpha} y^{1 / 2-\beta} z^{1 / 2-\gamma} J_{1 / 2-\alpha}\left(\sigma_{n} x\right) J_{1 / 2-\gamma}\left(\delta_{m} z\right) \times \\
\times\left[a_{n m} I_{1 / 2-\beta}\left(\sqrt{\lambda_{n m}} y\right)+b_{n m} K_{1 / 2-\beta}\left(\sqrt{\lambda_{n m}} y\right)\right], \\
u_{n m}^{-}(x, y, z)=x^{1 / 2-\alpha}(-y)^{1 / 2-\beta} z^{1 / 2-\gamma} J_{1 / 2-\alpha}\left(\sigma_{n} x\right) J_{1 / 2-\gamma}\left(\delta_{m} z\right) \times \\
\times\left[-a_{n m} J_{1 / 2-\beta}\left(\sqrt{\lambda_{n m}}(-y)\right)+b_{n m} \bar{Y}_{1 / 2-\beta}\left(\sqrt{\lambda_{n m}}(-y)\right)\right] .
\end{gathered}
$$

Постоянные $a_{n m}$ и $b_{n m}$ находим из требования, что решение $(34)$ должно удовлетворять граничным условиям (4) и (5). Сначала, подставляя его в условия (4), получим

$$
\sum_{n=1}^{\infty} J_{1 / 2-\alpha}\left(\sigma_{n} x\right) \varphi_{n}(z)=x^{\alpha-1 / 2} b^{\beta-1 / 2} z^{\gamma-1 / 2} f(x, z)
$$

где

$\varphi_{n}(z)=\sum_{m=1}^{\infty} J_{1 / 2-\gamma}\left(\delta_{m} z\right)\left[a_{n m} I_{1 / 2-\beta}\left(\sqrt{\lambda_{n m}} b\right)+b_{n m} K_{1 / 2-\beta}\left(\sqrt{\lambda_{n m}} b\right)\right]$

Ряды (35) и (36) - ряды Фурье-Бесселя [15, §18.1], поэтому

$$
\begin{gathered}
\varphi_{n}(z)=\frac{2}{J_{3 / 2-\alpha}^{2}\left(\sigma_{n}\right)} \int_{0}^{1} t^{1 / 2+\alpha} b^{\beta-1 / 2} z^{\gamma-1 / 2} f(t, z) J_{1 / 2-\alpha}\left(\sigma_{n} t\right) d t \\
a_{n m} I_{1 / 2-\beta}\left(\sqrt{\lambda_{n m}} b\right)+b_{n m} K_{1 / 2-\beta}\left(\sqrt{\lambda_{n m}} b\right)=f_{n m}
\end{gathered}
$$

где

$$
\begin{aligned}
f_{n m}= & \frac{4 b^{\beta-1 / 2}}{\left[J_{3 / 2-\alpha}\left(\sigma_{n}\right) J_{3 / 2-\gamma}\left(\delta_{m}\right)\right]^{2}} \times \\
& \times \int_{0}^{1} \int_{0}^{1} t^{1 / 2+\alpha} s^{1 / 2+\gamma} J_{1 / 2-\alpha}\left(\sigma_{n} t\right) J_{1 / 2-\gamma}\left(\delta_{m} s\right) f(t, s) d t d s .
\end{aligned}
$$


На основании формул дифференцирования бесселевых функций $[15, \S 3.2]$

$$
\frac{d}{d x}\left[x^{\nu} J_{\nu}(x)\right]=x^{\nu} J_{\nu-1}(x)
$$

коэффициенты $f_{n m}$ представим в виде

$$
\begin{aligned}
f_{n m}=\frac{4 b^{\beta-1 / 2}}{\sigma_{n} \delta_{m}\left[J_{3 / 2-\alpha}\left(\sigma_{n}\right) J_{3 / 2-\gamma}\left(\delta_{m}\right)\right]^{2}} \int_{0}^{1} \int_{0}^{1} f(t, s) t^{2 \alpha-1} s^{2 \gamma-1} \times \\
\quad \times \frac{d}{d t}\left[t^{3 / 2-\alpha} J_{3 / 2-\alpha}\left(\sigma_{n} t\right)\right] \frac{d}{d s}\left[s^{3 / 2-\gamma} J_{3 / 2-\gamma}\left(\delta_{m} s\right)\right] d t d s .
\end{aligned}
$$

Интегрируя по частям интеграл в (38), получим

$$
\begin{gathered}
f_{n m}=\frac{4 b^{\beta-1 / 2}}{\sigma_{n} \delta_{m}\left[J_{3 / 2-\alpha}\left(\sigma_{n}\right) J_{3 / 2-\gamma}\left(\delta_{m}\right)\right]^{2}}\left\{J_{3 / 2-\alpha}\left(\sigma_{n}\right) J_{3 / 2-\gamma}\left(\delta_{m}\right) f(1,1)-\right. \\
-J_{3 / 2-\alpha}\left(\sigma_{n}\right) \int_{0}^{1} s^{3 / 2-\gamma} J_{3 / 2-\gamma}\left(\delta_{m} s\right) \frac{d}{d s}\left[f(1, s) s^{2 \gamma-1}\right] d s- \\
-J_{3 / 2-\gamma}\left(\delta_{m}\right) \int_{0}^{1} t^{3 / 2-\alpha} J_{3 / 2-\alpha}\left(\sigma_{n} t\right) \frac{d}{d t}\left[f(t, 1) t^{2 \alpha-1}\right] d t+ \\
+\int_{0}^{1} \int_{0}^{1} t^{3 / 2-\alpha} s^{3 / 2-\gamma} J_{3 / 2-\alpha}\left(\sigma_{n} t\right) J_{3 / 2-\gamma}\left(\delta_{m} s\right) \times \\
\left.\times \frac{\partial^{2}}{\partial t \partial s}\left[t^{2 \alpha-1} s^{2 \gamma-1} f(t, s)\right] d t d s\right\} .
\end{gathered}
$$

В силу условий задачи D справедливо равенство

$$
f(1, z)=f(x, 1)=0, \quad 0 \leqslant z \leqslant 1, \quad 0 \leqslant x \leqslant 1 .
$$

Тогда формула (39) примет вид

$$
\begin{aligned}
& f_{n m}=\frac{4 b^{\beta-1 / 2}}{\sigma_{n} \delta_{m}\left[J_{3 / 2-\alpha}\left(\sigma_{n}\right) J_{3 / 2-\gamma}\left(\delta_{m}\right)\right]^{2}} \times \\
& \times \int_{0}^{1} \int_{0}^{1} t^{3 / 2-\alpha} s^{3 / 2-\gamma} J_{3 / 2-\alpha}\left(\sigma_{n} t\right) J_{3 / 2-\gamma}\left(\delta_{m} s\right) \times \\
& \times \frac{\partial^{2}}{\partial t \partial s}\left[t^{2 \alpha-1} s^{2 \gamma-1} f(t, s)\right] d t d s .
\end{aligned}
$$

Из последнего выражения после интегрирования по частям имеем

$$
\begin{aligned}
& f_{n m}=\frac{4 b^{\beta-1 / 2}}{\sigma_{n}^{2} \delta_{m}^{2}\left[J_{3 / 2-\alpha}\left(\sigma_{n}\right) J_{3 / 2-\gamma}\left(\delta_{m}\right)\right]^{2}} \times \\
& \quad \times\left\{J_{5 / 2-\alpha}\left(\sigma_{n}\right) J_{5 / 2-\gamma}\left(\delta_{m}\right) \frac{\partial^{2}}{\partial t \partial s}\left[t^{2 \alpha-1} s^{2 \gamma-1} f(t, s)\right]_{\substack{t=1 \\
s=1}}-\right.
\end{aligned}
$$




$$
\begin{gathered}
-J_{5 / 2-\gamma}\left(\delta_{m}\right) \int_{0}^{1} t^{5 / 2-\alpha} J_{5 / 2-\alpha}\left(\sigma_{n} t\right) \frac{\partial}{\partial t}\left[t^{-1} \frac{\partial^{2}}{\partial t \partial s}\left[t^{2 \alpha-1} s^{2 \gamma-1} f(t, s)\right]\right] s=1 d t- \\
-J_{5 / 2-\alpha}\left(\sigma_{n}\right) \int_{0}^{1} s^{5 / 2-\gamma} J_{5 / 2-\gamma}\left(\delta_{m} s\right) \frac{\partial}{\partial s}\left[s^{-1} \frac{\partial^{2}}{\partial t \partial s}\left[t^{2 \alpha-1} s^{2 \gamma-1} f(t, s)\right]\right]_{t=1} d s+ \\
+\int_{0}^{1} \int_{0}^{1} t^{5 / 2-\alpha} s^{5 / 2-\gamma} J_{5 / 2-\alpha}\left(\sigma_{n} t\right) J_{5 / 2-\gamma}\left(\delta_{m} s\right) \times \\
\left.\times \frac{\partial^{2}}{\partial t \partial s}\left[t^{-1} s^{-1} \frac{\partial^{2}}{\partial t \partial s}\left[t^{2 \alpha-1} s^{2 \gamma-1} f(t, s)\right]\right] d t d s\right\} .
\end{gathered}
$$

Отсюда следует, что если функция $f(x, z)$ удовлетворяет условиям

$$
\begin{gathered}
\tilde{f}(x, z)=\left(\partial^{2} / \partial x \partial z\right)\left[x^{2 \alpha-1} z^{2 \gamma-1} f(x, z)\right] \in C([0,1] \times[0,1]), \\
\lim _{x \rightarrow 1} \tilde{f}(x, z)=\lim _{z \rightarrow 1} \tilde{f}(x, z)=0,
\end{gathered}
$$

то функции $f_{n m}$ определяются формулами

$$
\begin{aligned}
& f_{n m}=\frac{4 b^{\beta-1 / 2}}{\sigma_{n}^{2} \delta_{m}^{2}\left[J_{3 / 2-\alpha}\left(\sigma_{n}\right) J_{3 / 2-\gamma}\left(\delta_{m}\right)\right]^{2}} \times \\
& \times \int_{0}^{1} \int_{0}^{1} t^{5 / 2-\alpha} s^{5 / 2-\gamma} J_{5 / 2-\alpha}\left(\sigma_{n} t\right) J_{5 / 2-\gamma}\left(\delta_{m} s\right) \times \\
& \times \frac{\partial^{2}}{\partial t \partial s}\left[(t s)^{-1} \tilde{f}(t, s)\right] d t d s .
\end{aligned}
$$

Интегрируя по частям последнее выражение, получим

$$
\begin{aligned}
f_{n m} & =\frac{4 b^{\beta-1 / 2}}{\sigma_{n}^{3} \delta_{m}^{3}\left[J_{3 / 2-\alpha}\left(\sigma_{n}\right) J_{3 / 2-\gamma}\left(\delta_{m}\right)\right]^{2}} \times \\
& \times\left\{J_{7 / 2-\alpha}\left(\sigma_{n}\right) J_{7 / 2-\gamma}\left(\delta_{m}\right) \frac{\partial^{2}}{\partial t \partial s}\left[(t s)^{-1} \tilde{f}(t, s)\right]_{t=1}-\right. \\
& -J_{7 / 2-\gamma}\left(\delta_{m}\right) \int_{0}^{1} t^{7 / 2-\alpha} J_{7 / 2-\alpha}\left(\sigma_{n} t\right) \frac{\partial}{\partial t}\left[t^{-1} \frac{\partial^{2}}{\partial t \partial s}\left[(t s)^{-1} \tilde{f}(t, s)\right]\right]_{s=1} d t- \\
& -J_{7 / 2-\alpha}\left(\sigma_{n}\right) \int_{0}^{1} s^{7 / 2-\gamma} J_{7 / 2-\gamma}\left(\delta_{m} s\right) \frac{\partial}{\partial s}\left[s^{-1} \frac{\partial^{2}}{\partial t \partial s}\left[(t s)^{-1} \tilde{f}(t, s)\right]\right]_{t=1} d s+ \\
+ & \left.\int_{0}^{1} \int_{0}^{1} t^{7 / 2-\alpha} s^{7 / 2-\gamma} J_{7 / 2-\alpha}\left(\sigma_{n} t\right) J_{7 / 2-\gamma}\left(\delta_{m} s\right) \times \frac{\partial^{2}}{\partial t \partial s}\left[(t s)^{-1} \frac{\partial^{2}}{\partial t \partial s}\left[(t s)^{-1} \tilde{f}(t, s)\right]\right] d t d s\right\} .
\end{aligned}
$$

Если здесь потребовать выполнения условий

$$
\begin{gathered}
\tilde{f}_{0}(x, z)=\left(\partial^{2} / \partial x \partial z\right)\left[(x z)^{-1} \tilde{f}(x, z)\right] \in C([0,1] \times[0,1]), \\
\lim _{x \rightarrow 1} \tilde{f}_{0}(x, z)=\lim _{z \rightarrow 1} \tilde{f}_{0}(x, z)=0
\end{gathered}
$$


то $f_{n m}$ будет иметь вид

$$
\begin{aligned}
f_{n m}=\frac{4 b^{\beta-1 / 2}}{\sigma_{n}^{3} \delta_{m}^{3}\left[J_{3 / 2-\alpha}\left(\sigma_{n}\right) J_{3 / 2-\gamma}\left(\delta_{m}\right)\right]^{2}} \times & \\
\times \int_{0}^{1} \int_{0}^{1} t^{7 / 2-\alpha} s^{7 / 2-\gamma} J_{7 / 2-\alpha}\left(\sigma_{n} t\right) J_{7 / 2-\gamma}\left(\delta_{m} s\right) \times & \\
& \times \frac{\partial^{2}}{\partial t \partial s}\left[(t s)^{-1} \tilde{f}_{0}(t, s)\right] d t d s=\frac{\tilde{f}_{n m}}{\sigma_{n}^{3} \delta_{m}^{3}} .
\end{aligned}
$$

В силу этого равенства формула (37) принимает вид

$$
a_{n m} I_{1 / 2-\beta}\left(\sqrt{\lambda_{n m}} b\right)+b_{n m} K_{1 / 2-\beta}\left(\sqrt{\lambda_{n m}} b\right)=\frac{\tilde{f}_{n m}}{\sigma_{n}^{3} \delta_{m}^{3}} .
$$

Аналогично, подставляя (34) в условия (5) и требуя выполнения следующих групп условий:

$$
\begin{gathered}
g(1, z)=g(x, 1)=0, \\
\tilde{g}(x, z)=\left(\partial^{2} / \partial x \partial z\right)\left[x^{2 \alpha-1} z^{2 \gamma-1} g(x, z)\right] \in C([0,1] \times[0,1]), \\
\lim _{x \rightarrow 1} \tilde{g}(x, z)=\lim _{z \rightarrow 1} \tilde{g}(x, z)=0 \\
\tilde{g}_{0}(x, z)=\left(\partial^{2} / \partial x \partial z\right)\left[(x z)^{-1} \tilde{g}(x, z)\right] \in C([0,1] \times[0,1]), \\
\lim _{x \rightarrow 1} \tilde{g}_{0}(x, z)=\lim _{z \rightarrow 1} \tilde{g}_{0}(x, z)=0,
\end{gathered}
$$

получим соотношение

$$
-a_{n m} J_{1 / 2-\beta}\left(\sqrt{\lambda_{n m}} a\right)+b_{n m} \bar{Y}_{1 / 2-\beta}\left(\sqrt{\lambda_{n m}} a\right)=\frac{\tilde{g}_{n m}}{\sigma_{n}^{3} \delta_{m}^{3}},
$$

где

$$
\begin{aligned}
& \frac{\tilde{g}_{n m}}{\sigma_{n}^{3} \delta_{m}^{3}}=\frac{4 a^{\beta-1 / 2}}{\sigma_{n}^{3} \delta_{m}^{3}\left[J_{3 / 2-\alpha}\left(\sigma_{n}\right) J_{3 / 2-\gamma}\left(\delta_{m}\right)\right]^{2}} \times \\
& \times \int_{0}^{1} \int_{0}^{1} t^{7 / 2-\alpha} s^{7 / 2-\gamma} J_{7 / 2-\alpha}\left(\sigma_{n} t\right) J_{7 / 2-\gamma}\left(\delta_{m} s\right) \times \\
& \times \frac{\partial^{2}}{\partial t \partial s}\left\{t^{-1} s^{-1} \frac{\partial^{2}}{\partial t \partial s}\left[t^{-1} s^{-1} \frac{\partial^{2}}{\partial t \partial s}\left[t^{2 \alpha-1} s^{2 \gamma-1} g(t, s)\right]\right]\right\} d t d s=g_{n m} .
\end{aligned}
$$

Далее, разрешая систему линейных алгебраических уравнений (43), (45) относительно $a_{n m}$ и $b_{n m}$, получим

$$
\begin{aligned}
a_{n m} & =\frac{\tilde{f}_{n m} \bar{Y}_{1 / 2-\beta}\left(\sqrt{\lambda_{n m}} a\right)-\tilde{g}_{n m} K_{1 / 2-\beta}\left(\sqrt{\lambda_{n m}} b\right)}{\sigma_{n}^{3} \delta_{m}^{3} \Delta_{n m}(a, b)}, \\
b_{n m} & =\frac{\tilde{f}_{n m} J_{1 / 2-\beta}\left(\sqrt{\lambda_{n m}} a\right)+\tilde{g}_{n m} I_{1 / 2-\beta}\left(\sqrt{\lambda_{n m}} b\right)}{\sigma_{n}^{3} \delta_{m}^{3} \Delta_{n m}(a, b)} .
\end{aligned}
$$


Подставляя найденные значения $a_{n m}$ и $b_{n m}$ в $(34)$, получим формальное решение задачи D.

Теперь докажем, что функция (34) является решением задачи D. Для этого вначале докажем равномерную сходимость рядов в (34) в $\bar{\Omega}$.

Известно [16, форм. (32.31) и (32.33)], что для цилиндрических функций при $\xi \rightarrow \infty$ имеют место следующие асимптотические формулы:

$$
\begin{gathered}
J_{\nu}(\xi)=\sqrt{\frac{2}{\pi \xi}}\left[\cos \left(\xi-\frac{\nu \pi}{2}-\frac{\pi}{4}\right)+O\left(\xi^{-1}\right)\right], \\
I_{\nu}(\xi)=\frac{e^{\xi}}{\sqrt{2 \pi \xi}}\left[1+O\left(\xi^{-1}\right)\right], \quad K_{\nu}(\xi)=\sqrt{\frac{\pi}{2 \xi}} e^{-\xi}\left[1+O\left(\xi^{-1}\right)\right] .
\end{gathered}
$$

В этих формулах запись $O\left(\xi^{-1}\right)[17, \S 2]$ означает такую величину, отношение которой к $\xi^{-1}$ при беспредельном возрастании $\xi$ остается меньше некоторой постоянной, при этом справедливы следующие формулы при $\xi \rightarrow \infty$ :

$$
\begin{gathered}
f(\xi)=O(f(\xi)), \\
O(O(f(\xi)))=O(f(\xi)), \\
O(f(\xi)) O(g(\xi))=O(f(\xi) g(\xi)), \\
O(f(\xi))+O(g(\xi))=O(|f(\xi)|+|g(\xi)|), \\
C \cdot O(f(\xi))=O(f(\xi)), \\
O(f(\xi) g(\xi))=f(\xi) O(g(\xi)), \\
O\left(f^{2}(\xi)\right)=O(f(\xi))^{2}, \\
O\left(\xi^{-2}\right)=O\left(\xi^{-1}\right),
\end{gathered}
$$

где $f$ - произвольная функция.

Применяя последовательно формулы (48), (52), (50), (55), (51) и (53) к равенству (46), а формулы (48), (50), (55) и (52) к равенствам (47), получим асимптотические формулы

$$
\begin{gathered}
J_{\nu}(\xi)=O\left(\xi^{-1 / 2}\right) \\
I_{\nu}(\xi)=O\left(\xi^{-1 / 2}\right) e^{\xi}, \quad K_{\nu}(\xi)=O\left(\xi^{-1 / 2}\right) e^{-\xi}
\end{gathered}
$$

Известно [15, с.653], что если $f(t) \in C[0,1]$, то при $\xi \rightarrow \infty$ справедлива формула

$$
\int_{0}^{1} f(t) t J_{\nu}(\xi t) d t=O\left(\xi^{-3 / 2}\right) .
$$

С помощью асимптотических формул (56)-(58) и формул (48)-(55) нетрудно показать, что при $n \rightarrow \infty$ и $m \rightarrow \infty$ имеют место следующие формулы:

$$
\begin{gathered}
\tilde{f}_{n m}=O\left(\left(\sigma_{n} \delta_{m}\right)^{-1 / 2}\right), \quad \tilde{g}_{n m}=O\left(\left(\sigma_{n} \delta_{m}\right)^{-1 / 2}\right), \\
\bar{Y}_{1 / 2-\beta}\left(\sqrt{\lambda_{n m}} a\right)=O\left(\lambda_{n m}^{-1 / 4}\right), \quad K_{1 / 2-\beta}\left(\sqrt{\lambda_{n m}} b\right)=O\left(\lambda_{n m}^{-1 / 4}\right) e^{-\sqrt{\lambda_{n m}} b}, \\
J_{1 / 2-\beta}\left(\sqrt{\lambda_{n m}} a\right)=O\left(\lambda_{n m}^{-1 / 4}\right), \quad I_{1 / 2-\beta}\left(\sqrt{\lambda_{n m}} b\right)=O\left(\lambda_{n m}^{-1 / 4}\right) e^{\sqrt{\lambda_{n m}} b}, \\
\Delta_{n m}(a, b)=O\left(\lambda_{n n m}^{-1 / 2}\right) e^{\sqrt{\lambda_{n m}} b}, \\
a_{n m}=O\left(\frac{\lambda_{n m}^{1 / 4}}{\left(\sigma_{n} \delta_{m}\right)^{7 / 2}}\right) e^{-\sqrt{\lambda_{n m}} b}, \quad b_{n m}=O\left(\frac{\lambda_{n m}^{1 / 4}}{\left(\sigma_{n} \delta_{m}\right)^{7 / 2}}\right) .
\end{gathered}
$$


Из асимптотических формул (56), (57), (59) следует, что если $0 \leqslant x \leqslant 1$, $0 \leqslant z \leqslant 1,-a \leqslant y \leqslant b$, то при $n \rightarrow \infty, m \rightarrow \infty$ имеют место формулы

$$
u_{n m}^{+}(x, y, z)=O\left(\left(\sigma_{n} \delta_{m}\right)^{-4}\right), \quad u_{n m}^{-}(x, y, z)=O\left(\left(\sigma_{n} \delta_{m}\right)^{-4}\right) .
$$

Отсюда следует, что для членов рядов в (34) в $\bar{\Omega}$ справедливы следующие оценки:

$$
\left|u_{n m}^{+}(x, y, z)\right| \leqslant C_{1}\left(\sigma_{n} \delta_{m}\right)^{-4}, \quad\left|u_{n m}^{-}(x, y, z)\right| \leqslant C_{2}\left(\sigma_{n} \delta_{m}\right)^{-4},
$$

где $C_{1}, C_{2}$ - некоторые положительные постоянные.

Тогда в силу признака Вейерштрасса $[18, \S 430]$ ряды в (34) сходятся абсолютно и равномерно в $\bar{\Omega}$ и, следовательно, $u(x, y, z) \in C(\bar{\Omega})$.

Теперь докажем, что $u_{x x}(x, y, z) \in C\left(\Omega^{+} \cup \Omega^{-}\right)$. Дважды почленно продифференцируем ряд (34) по аргументу $x$ :

$$
u_{x x}(x, y, z)= \begin{cases}\sum_{n=1}^{\infty} \sum_{m=1}^{\infty}\left(u_{n m}^{+}(x, y, z)\right)_{x x}, & y>0 \\ \sum_{n=1}^{\infty} \sum_{m=1}^{\infty}\left(u_{n m}^{-}(x, y, z)\right)_{x x}, & y<0\end{cases}
$$

где

$$
\begin{gathered}
\left(u_{n m}^{+}(x, y, z)\right)_{x x}=\left[\sigma_{n} x^{-1 / 2-\alpha} J_{-1 / 2-\alpha}\left(\sigma_{n} x\right)+\sigma_{n}^{2} x^{1 / 2-\alpha} J_{-3 / 2-\alpha}\left(\sigma_{n} x\right)\right] \times \\
\times y^{1 / 2-\beta} z^{1 / 2-\gamma} J_{1 / 2-\gamma}\left(\delta_{m} z\right)\left[a_{n m} I_{1 / 2-\beta}\left(\sqrt{\lambda_{n m}} y\right)+b_{n m} K_{1 / 2-\beta}\left(\sqrt{\lambda_{n m}} y\right)\right] \\
\left(u_{n m}^{-}(x, y, z)\right)_{x x}=\left[\sigma_{n} x^{-1 / 2-\alpha} J_{-1 / 2-\alpha}\left(\sigma_{n} x\right)+\sigma_{n}^{2} x^{1 / 2-\alpha} J_{-3 / 2-\alpha}\left(\sigma_{n} x\right)\right] \times \\
\times(-y)^{1 / 2-\beta} z^{1 / 2-\gamma} J_{1 / 2-\gamma}\left(\delta_{m} z\right) \times \\
\times\left[-a_{n m} J_{1 / 2-\beta}\left(\sqrt{\lambda_{n m}}(-y)\right)+b_{n m} \bar{Y}_{1 / 2-\beta}\left(\sqrt{\lambda_{n m}}(-y)\right)\right] .
\end{gathered}
$$

Применяя асимптотические формулы $(56),(57)$ и $(59)$, при $(x, y, z) \in \Omega^{+} \cup \Omega^{-}$ и $n \rightarrow \infty, m \rightarrow \infty$ получим

$$
\left(u_{n m}^{+}(x, y, z)\right)_{x x}=O\left(\left(\sigma_{n}^{2} \delta_{m}^{4}\right)^{-1}\right), \quad\left(u_{n m}^{-}(x, y, z)\right)_{x x}=O\left(\left(\sigma_{n}^{2} \delta_{m}^{4}\right)^{-1}\right) .
$$

Отсюда следует, что для членов рядов в $(60)$ в $\Omega^{+} \cup \Omega^{-}$имеют место следующие оценки:

$$
\left|\left(u_{n m}^{+}(x, y, z)\right)_{x x}\right| \leqslant C_{3}\left(\sigma_{n}^{2} \delta_{m}^{4}\right)^{-1}, \quad\left|\left(u_{n m}^{-}(x, y, z)\right)_{x x}\right| \leqslant C_{4}\left(\sigma_{n}^{2} \delta_{m}^{4}\right)^{-1},
$$

где $C_{3}, C_{4}$ - некоторые положительные постоянные.

Из полученных оценок следует, что ряды из (60) мажорируются сходящимися числовыми рядами, откуда следует, что ряды из (60) абсолютно и равномерно сходятся в $\Omega^{+} \cup \Omega^{-}$. Следовательно, $u_{x x}(x, y, z) \in C\left(\Omega^{+} \cup \Omega^{-}\right)$.

Аналогично доказывается, что $u_{z z}(x, y, z) \in C\left(\Omega^{+} \cup \Omega^{-}\right)$. 
Далее покажем, что $u_{y y}(x, y, z) \in C\left(\Omega^{+} \cup \Omega^{-}\right)$. Продифференцируем дважды (34) по аргументу $y$ :

$$
u_{y y}(x, y, z)= \begin{cases}\sum_{n=1}^{\infty} \sum_{m=1}^{\infty}\left(u_{n m}^{+}(x, y, z)\right)_{y y}, & y>0 \\ \sum_{n=1}^{\infty} \sum_{m=1}^{\infty}\left(u_{n m}^{-}(x, y, z)\right)_{y y}, & y<0\end{cases}
$$

где

$$
\begin{gathered}
\left(u_{n m}^{+}(x, y, z)\right)_{y y}=x^{1 / 2-\alpha} z^{1 / 2-\gamma} J_{1 / 2-\alpha}\left(\sigma_{n} x\right) J_{1 / 2-\gamma}\left(\delta_{m} z\right) \times \\
\times\left\{\sqrt{\lambda_{n m}} y^{-1 / 2-\beta}\left[a_{n m} I_{-1 / 2-\beta}\left(\sqrt{\lambda_{n m}} y\right)-b_{n m} K_{-1 / 2-\beta}\left(\sqrt{\lambda_{n m}} y\right)\right]+\right. \\
\left.+\lambda_{n m} y^{1 / 2-\beta}\left[a_{n m} I_{-3 / 2-\beta}\left(\sqrt{\lambda_{n m}} y\right)+b_{n m} K_{-3 / 2-\beta}\left(\sqrt{\lambda_{n m}} y\right)\right]\right\} \\
\left(u_{n m}^{-}(x, y, z)\right)_{y y}=x^{1 / 2-\alpha} z^{1 / 2-\gamma} J_{1 / 2-\alpha}\left(\sigma_{n} x\right) J_{1 / 2-\gamma}\left(\delta_{m} z\right) \times \\
\times\left\{\sqrt { \lambda _ { n m } } ( - y ) ^ { - 1 / 2 - \beta } \left[-a_{n m} J_{-1 / 2-\beta}\left(\sqrt{\lambda_{n m}}(-y)\right)+\right.\right. \\
+\frac{b_{n m} \pi}{2 \cos (\beta \pi)}\left[J_{-1 / 2-\beta}\left(\sqrt{\lambda_{n m}}(-y)\right)-J_{1 / 2+\beta}\left(\sqrt{\lambda_{n m}}(-y)\right)\right]+ \\
+\lambda_{n m}(-y)^{1 / 2-\beta}\left[-a_{n m} J_{-3 / 2-\beta}\left(\sqrt{\lambda_{n m}}(-y)\right)+\right. \\
\left.\left.+b_{n m} \bar{Y}_{(3 / 2)+\beta}\left(\sqrt{\lambda_{n m}}(-y)\right)\right]\right\} .
\end{gathered}
$$

Применяя асимптотические формулы (56), (48), (57), (59) и (50), при $(x, y, z) \in \Omega^{+} \cup \Omega^{-}$и $n \rightarrow \infty, m \rightarrow \infty$ имеем

$$
\left(u_{n m}^{+}(x, y, z)\right)_{y y}=O\left(\frac{\lambda_{n m}}{\left(\sigma_{n} \delta_{m}\right)^{4}}\right), \quad\left(u_{n m}^{-}(x, y, z)\right)_{y y}=O\left(\frac{\lambda_{n m}}{\left(\sigma_{n} \delta_{m}\right)^{4}}\right) .
$$

Отсюда следует, что для членов рядов из (61) в $\Omega^{+} \cup \Omega^{-}$имеют место следующие оценки:

$$
\left|\left(u_{n m}^{+}(x, y, z)\right)_{y y}\right|=C_{5} \frac{\lambda_{n m}}{\left(\sigma_{n} \delta_{m}\right)^{4}}, \quad\left|\left(u_{n m}^{-}(x, y, z)\right)_{y y}\right|=C_{6} \frac{\lambda_{n m}}{\left(\sigma_{n} \delta_{m}\right)^{4}},
$$

где $C_{5}, C_{6}$ - некоторые положительные постоянные.

Из этих оценок следует, что ряды из (61) мажорируются сходящимися числовыми рядами, откуда следует, что ряды из (61) абсолютно и равномерно сходятся в $\Omega^{+} \cup \Omega^{-}$. Следовательно, $u_{y y}(x, y, z) \in C\left(\Omega^{+} \cup \Omega^{-}\right)$.

Таким образом, доказана следующая теорема.

Теорема 2. Пусть выполнены условия (40), (41), (42) и (44). Тогда решение задачи Дирихле существует, единственно и определяется формулами (34).

В заключение отметим, что задача D исследуется аналогично и в том случае, когда некоторые или все параметры $\alpha, \beta$ и $\gamma$ равны нулю.

Конкурирующие интересы. Заявляем, что в отношении авторства и публикации этой статьи конфликта интересов не имеем. 
Авторский вклад и ответственность. Все авторы принимали участие в разработке концепции статьи и в написании рукописи. Авторы несут полную ответственность за предоставление окончательной рукописи в печать. Окончательная версия рукописи была одобрена всеми авторами.

Финансирование. Исследование выполнялось без финансирования.

\section{Библиографический список}

1. Франкль Ф. И. О задачах С. А. Чаплыгина для смешанных до- и сверхзвуковых течений // Изв. АН СССР. Сер. матем., 1945. Т. 9, № 2. С. 121-143.

2. Бицадзе А. В. Некорректность задачи Дирихле для уравнений смешанного типа // ДАН CCCP, 1953. T. 122 , № 2. C. 167-170.

3. Хачев М. М. Первая краевая задача для линейных уравнений смешанного типа. Нальчик: Эльбрус, 1998. 168 с.

4. Солдатов А. П. К теории уравнений смешанного типа/ Соврем. мат. и ее прил., Т. 10, Труды международной конференции по динамическим системам и дифференциальным уравнениям (Суздаль, 1-6 июля 2002 г.) Часть 4. Тбилиси: Институт кибернетики Академии наук Грузии, 2003. С. 153-162.

5. Сабитов К. Б. Задача Дирихле для уравнений смешанного типа в прямоугольной области // Докл. РАН, 2007. Т. 413, № 1. С. 23-26.

6. Сабитов К. Б., Сулейманова А. Х. Задача Дирихле для уравнения смешанного типа второго рода в прямоугольной области // Изв. вузов. Матем., 2007. № 4. С. 45-53.

7. Сабитов К. Б., Вагапова Э. В. Задача Дирихле для уравнения смешанного типа с двумя линиями вырождения в прямоугольной области // Дифферени. уравнения, 2013. Т. 49, № 1. С. $68-78$.

8. Хайруллин Р. С. К задаче Дирихле для уравнения смешанного типа второго рода с сильным вырождением // Дифферени. уравнения, 2013. Т. 49, № 4. С. 528-534. doi: 10 . 1134/S0374064113040122.

9. Сафина Р. М. Задача Дирихле для уравнения Пулькина в прямоугольной области // Вестн. СамГУ. Естественнонаучн. сер., 2014. №10(121). С. 91-101.

10. Zhang K, Li Y. On Dirichlet problem of Tricomi-type equation in rectangular domains // J. Nanjing Norm. Univ., Nat. Sci. Ed., 2016. vol.39, no.1. pp. 29-35. doi:10.3969/j. issn.1001-4616.2016.01.005.

11. Нахушев А. М. Критерий единственности задачи Дирихле для уравнения смешанного типа в цилиндрической области // Дифферени. уравнения, 1970. Т. 6, №1. С. 190-191.

12. Сафина Р. М. Задача Дирихле с осевой симметрией для уравнения смешанного $B$ эллиптико-B-гиперболического типа с характеристическим вырождением // Becтник Татарского государственного гуманитарно-педагогического университета, 2010. № 4 . C. 63-69.

13. Алдашев С. А. Критерий однозначной разрешимости спектральной задачи Дирихле в цилиндрической области для многомерного уравнения Лаврентьева-Бицадзе // Изв. вузов. Матем., 2011. № 4. С. 3-7.

14. Сафина Р. М. Критерий единственности решения задачи Дирихле с осевой симметрией для трехмерного уравнения смешанного типа с оператором Бесселя // Изв. вузов. Матем., 2014. №6. С. 78-83.

15. Watson G. N. A Treatise on the Theory of Bessel Functions. Cambridge: Cambridge University Press, 1922.

16. Кузнецов Д. С. Специальные функции. М.: Высшая школа, 1962. 448 с.

17. Olver F. W. J. Introduction to Asymptotic Analysis/ Introduction to Asymptotics and Special Functions. New York: Academic Press, Inc., 1974. pp. 1-30. doi: 10.1016/ b978-0-12-525856-2.50005-x.

18. Фихтенгольц Г. М. Курс дифференциального и интегрального исчисления. Т. 2. М.: Наука, 1969. 800 c. 
MSC: 35M10, 35M12

\title{
The Dirichlet problem for a three-dimensional equation of mixed type with three singular coefficients
}

\author{
A. K. Urinov, K. T.Karimov \\ Fergana State University, \\ 19, Murabbiylar st., Fergana, 712000, Uzbekistan.
}

\begin{abstract}
We study the Dirichlet problem in a parallelepiped for a three-dimensional equation of mixed type with three singular coefficients. Separation of variables with Fourier series and spectral analysis are used to investigate this problem. Two one-dimensional spectral problems are obtained for the possed problem using the Fourier method. On the basis of the completeness property of the eigenfunction systems of these problems, the uniqueness theorem is proved. The solution of the problem is constructed as the sum of a double Fourier-Bessel series. In justification of the uniform convergence of the series constructed, asymptotic estimates of the Bessel functions of the real and imaginary argument are used. On their basis, estimates are obtained for each member of the series. The estimates obtained made it possible to prove the convergence of the series and its derivatives up to the second order inclusive, and also the existence theorem in the class of regular solutions.
\end{abstract}

Keywords: Dirichlet problem, mixed-type equations, spectral method, uniqueness of solution, existence of solution.

Received: $19^{\text {th }}$ July, $2017 /$ Revised: $30^{\text {th }}$ November, $2017 /$ Accepted: $18^{\text {th }}$ December, $2017 /$ First online: $22^{\text {nd }}$ December, 2017

Competing interests. We declare that we have no conflicts of interests with the authorship and publication of this article.

Authors' contributions and responsibilities. Each author has participated in the article concept development and in the manuscript writing. The authors are absolutely responsible for submitting the final manuscript in print. Each author has approved the final version of manuscript.

\section{Research Article}

○ () The content is published under the terms of the Creative Commons Attribution 4.0 International License (http://creativecommons.org/licenses/by/4.0/)

Please cite this article in press as:

Urinov A. K., Karimov K. T. The Dirichlet problem for a three-dimensional equation of mixed type with three singular coefficients, Vestn. Samar. Gos. Tekhn. Univ., Ser. Fiz.-Mat. Nauki [J. Samara State Tech. Univ., Ser. Phys. Math. Sci.], 2017, vol. 21, no. 4, pp. 665-683. doi: 10.14498/vsgtu1559 (In Russian).

\section{Authors' Details:}

Akhmadjon K. Urinov (1) http://orcid.org/0000-0002-9586-1799

Dr. Phys. \& Math. Sci.; Professor; Dept. of Mathematical Analysis and Differential Equations; e-mail: urinovak@mail.ru

Kamoliddin T. Karimov (D) http://orcid.org/0000-0002-9098-4116

Cand. Phys. \& Math. Sci.; Doctoral Candidate; Dept. of Mathematical Analysis and Differential Equations; e-mail: karimovk80@mail.ru 
Funding. The research has not had any funding.

\section{References}

1. Frankl F. To the theory of the Laval nozzle, Izv. Akad. Nauk SSSR Ser. Mat., 1945, vol.9, no. 2, pp. 121-143 (In Russian).

2. Bitsadze A. V. The incorrectness of the Dirichlet problem for equations of mixed type, Dokl. Akad. Nauk SSSR, 1953, vol.122, no. 2, pp. 167-170 (In Russian).

3. Khachev M. M. Pervaia kraevaia zadacha dlia lineinykh uravnenii smeshannogo tipa [The First Boundary Value Problem for Linear Equations of Mixed Type]. Nalchik, Elbrus, 1998, 168 pp. (In Russian)

4. Soldatov A. P. On the theory of mixed-type equations, J. Math. Sci., 2005, vol. 129, no. 1, pp. 3670-3679. doi: 10.1007/s10958-005-0306-9.

5. Sabitov K. B. The Dirichlet problem for equations of mixed type in a rectangular domain, Dokl. Math., 2007, vol. 75, no. 2, pp. 193-196.

6. Sabitov K. B., Suleimanova A. Kh. The Dirichlet problem for a mixed-type equation of the second kind in a rectangular domain, Russian Math. (Iz. VUZ), 2007, vol.51, no. 4, pp. 42-50. doi : 10.3103/S1066369X07040068.

7. Sabitov K. B., Vagapova E. V. Dirichlet problem for an equation of mixed type with two degeneration lines in a rectangular domain, Differ. Equ., 2013, vol.49, no. 1, pp. 68-78. doi : 10.1134/S0012266113010072.

8. Khairullin R. S. On the Dirichlet problem for a mixed-type equation of the second kind with strong degeneration, Differ. Equ., 2013, vol.49, no.4, pp. 510-516. doi: 10.1134/ S0012266113040113.

9. Safina R. M. Dirichlet problem for Pulkin's equation in a rectangular domain, Vestnik SamGU. Estestvenno-Nauchnaya Ser., 2014, no. 10(121), pp. 91-101 (In Russian).

10. Zhang K, Li Y. On Dirichlet problem of Tricomi-type equation in rectangular domains, J. Nanjing Norm. Univ., Nat. Sci. Ed., 2016, vol.39, no.1, pp. 29-35. doi:10.3969/j. issn.1001-4616.2016.01.005.

11. Nakhushev A. M. A uniqueness condition of the Dirichlet problem for an equation of mixed type in a cylindrical domain, Differ. Uravn., 1970, vol. 6, no. 1, pp. 190-191 (In Russian).

12. Safina R. M. The Dirichlet Problem with Axial Symmetry for a Mixed B-ellipticB-hyperbolic Type with Characteristic Degeneration, Vestnik Tatarskogo GumanitarnoPedagogicheskogo Universiteta, 2010, no. 4(22), pp. 63-69 (In Russian).

13. Aldashev S. A. A criterion for the unique solvability of the dirichlet spectral problem in a cylindrical domain for a multidimensional Lavrent'ev-Bitsadze equation, Russian Math. (Iz. VUZ), 2011, vol. 55, no. 4, pp. 1-4. doi: 10.3103/S1066369X11040013.

14. Safina R. M. A criterion of uniqueness of a solution to the Dirichlet problem with the axial symmetry for the three-dimensional mixed type equation with the Bessel operator, Russian Math. (Iz. VUZ), 2014, vol. 58, no. 6, pp. 69-73. doi : 10.3103/S1066369X14060085.

15. Watson G. N. A Treatise on the Theory of Bessel Functions. Cambridge, Cambridge University Press, 1922.

16. Kuznetsov D. S. Spetsial'nye funktsii [Special Functions]. Moscow, Vysshaia shkola, 1962, 448 pp. (In Russian)

17. Olver F. W. J. Introduction to Asymptotic Analysis, In: Introduction to Asymptotics and Special Functions. New York, Academic Press, Inc., 1974, pp. 1-30. doi: 10.1016/ b978-0-12-525856-2.50005-x.

18. Fikhtengol'ts G. M. Kurs differentsial'nogo i integral'nogo ischisleniia [Course of Differential and Integral Calculus], vol. 2. Moscow, Nauka, 1969, 800 pp. (In Russian) 